\title{
Linear Dispersion Theory of Parallel Electromagnetic Modes for Regularized Kappa-Distributions
}

\author{
Edin Husidic, ${ }^{1,2, a)}$ Marian Lazar, ${ }^{1,2}$ Horst Fichtner, ${ }^{1,3}$ Klaus Scherer, ${ }^{1,3}$ and Patrick Astfalk ${ }^{4,5}$ \\ 1) Institut für Theoretische Physik, Lehrstuhl IV: Plasma-Astroteilchenphysik, Ruhr-Universität Bochum, D-44780 Bochum, \\ Germany \\ 2) Centre for Mathematical Plasma Astrophysics, 3001 Leuven Belgium \\ ${ }^{3)}$ Research Department, Plasmas with Complex Interactions, Ruhr-Universität Bochum, D-44780 Bochum, \\ Germany \\ 4) Max-Planck-Institut für Plasmaphysik, D-85748 Garching, Germany \\ 5) 2-36-6 Imado, Taito-ku, Tokyo-to, 111-0024, Japan
}

(Dated: 8 June 2020)

The velocity particle distributions measured in-situ in space plasmas deviate from Maxwellian (thermal) equilibrium, showing enhanced suprathermal tails which are well described by the standard Kappa-distribution (SKD). Despite its successful application, the SKD is frequently disputed due to a series of unphysical implications like diverging velocity moments, preventing a macroscopic description of the plasma. The regularized Kappa-distribution (RKD) has been introduced to overcome these limitations, but the dispersion properties of RKD-plasmas are not explored yet. In the present paper we compute the wavenumber dispersion of the frequency and damping or growth rates for the electromagnetic modes in plasmas characterized by the RKD. This task is accomplished by using the grid-based kinetic dispersion solver LEOPARD developed for arbitrary gyrotropic distributions [Ref.11]. By reproducing previous results obtained for the SKD and Maxwellian, we validate the functionality of the code. Furthermore, we apply the isotropic as well as the anisotropic RKDs to investigate stable electromagnetic electron-cyclotron (EMEC) and ion-cyclotron (EMIC) modes as well as temperature-anisotropy-driven instabilities, both for the case $T_{\perp} / T_{\|}>1$ (EMEC and EMIC instabilities) and for the case $T_{\perp} / T_{\|}<1$ (proton and electron firehose instabilities), where $\|$ and $\perp$ denote directions parallel and perpendicular to the local time-averaged magnetic field. Provided that the cutoff parameter $\alpha$ is small enough, the results show that the RKDs reproduce the dispersion curves of the SKD plasmas at both qualitative and quantitative levels. For higher values, however, physically significant deviation occurs.

PACS numbers: 52.35.-g, 52.35.Hr, 52.35.Qz

Credits and permissions: This article may be downloaded for personal use only. Any other use requires prior permission of the author and AIP Publishing. This article appeared in Phys. Plasmas 27, 042110 (2020) and may be found at https://doi.org/10.1063/1.5145181.

\section{INTRODUCTION}

The existence of nonthermal particle populations in the heliospheric plasma is well confirmed by various in-situ measurements. ${ }^{2+4}$ The velocity distributions of plasma particles deviate from thermal (Maxwellian) equilibrium, exhibiting nonthermal features like suptrathermal tails and temperature anisotropies, which one expects in general to be the case for any dilute and low-collisional plasma in space. $\frac{5}{5}$ Nonequilibrium particle populations present in space plasmas, in particular in the solar wind and terrestrial magnetosphere, can explain the enhanced fluctuations reported in these environments. Whenever a particle velocity distribution deviates from a Maxwellian, there is free energy available that can be accessed by linear eigenmodes of the system, if a certain threshold is crossed ${ }^{6}$ This can lead to an exponential growth of in- stabilities, entertaining the wave fluctuations that carry the energy throughout the plasma. ${ }^{7}$

A widely used tool to describe the solar wind particle distributions is the (isotropic) standard Kappa-distribution (SKD)

$$
f_{\mathrm{SKD}}(v)=\frac{n}{\pi^{3 / 2} \Theta^{3}} \frac{\Gamma(\kappa+1)}{\kappa^{3 / 2} \Gamma(\kappa-1 / 2)}\left(1+\frac{v^{2}}{\kappa \Theta^{2}}\right)^{-\kappa-1}
$$

with $v$ being the particle speed, $\Theta$ the most probable speed related to kinetic temperature $9-11, n$ the particle number density and the $\kappa$-parameter associated with the high-energy power-law decrease of the distribution. The SKD has originally been introduced by Olber ${ }^{[12]}$ and Vasyliūnas ${ }^{9}$ in 1968 to describe electron distributions in Earth's magnetosphere, and since then has been employed in the interplanetary environment, ${ }^{21813}$ in the interstellar and intergalactic medium,, 1415 and even applied in experimental physics $\frac{1617}{17}$ This power-law function shows enhanced high-energy tails, extending from a Maxwellian core, and turns (approximately) into the same Maxwellian for $\kappa \rightarrow \infty$. A generalization of the SKD is the standard Bi-Kappa-distribution (SBKD)

$$
\begin{aligned}
f_{\mathrm{SBKD}}(v)= & \frac{n}{\pi^{3 / 2} \Theta_{\|} \Theta_{\perp}^{2}} \frac{\Gamma(\kappa+1)}{\kappa^{3 / 2} \Gamma(\kappa-1 / 2)} \\
& \times\left(1+\frac{v_{\|}^{2}}{\kappa \Theta_{\|}^{2}}+\frac{v_{\perp}^{2}}{\kappa \Theta_{\perp}^{2}}\right)^{-\kappa-1}
\end{aligned}
$$


which has been regularly applied to describe plasmas with anisotropic distributions, $\frac{18119}{11}$ where $\|$ and $\perp$ denote, respectively, directions parallel and perpendicular to the local background magnetic field.

Despite their current involvement in a wide variety of applications in space plasmas, these standard models are still controversial in the plasma physics community, mainly due to the diverging velocity moments for low values of $\kappa$, which prevents a macroscopic description of these plasma systems. More exactly, the existence of moments of order $l$ impose the restriction $20121 \quad \kappa>(l+1) / 2$ on the $\kappa$-parameter. Indeed, the SKD is defined in terms of $\Theta$, which is directly related to the second order moment (i.e., kinetic temperature) that requires $\kappa>3 / 2$. Further unphysical implications of the SKD are mainly concerning the entropy of the physical system and the contribution of superluminal particles to macroscopic quantities ${ }^{[22[23]}$ Regarding the validity of the SKD in non-relativistic applications, as for any non-relativistic theory in order to be valid, the contribution from superluminal particles, i.e., particles with $v>c$, to macroscopic quantities (e.g., pressure) must be negligible. However, Scherer et al. ${ }^{23}$ showed that for values of $\kappa<2$ superluminal particles may contribute significantly to the pressure. The RKD offers an elegant and straightforward way to reduce these effects, which otherwise would have been solved by adopting a relativistic Kappa model. However, a relativistic Kappa does not solve the divergence of higher order moments but complicates very much the kinetic approach. In the solar wind (kinetic) temperatures of electrons and ions are nonrelativistic but comparable, meaning that ions have speeds much lower than electrons, such that the regularization applied for electrons to reduce the effects of their superluminal populations may not be necessary for the ions. Additionally, Gaelzer and Ziebell ${ }^{24}$ found that a small-gyroradius treatment, which is used in the study of kinetic Alfvén waves, is seriously compromised when employing the SKD. To fix these problematic features a regularized (isotropic) Kappa-distribution (RKD) has been introduced in the form 20

$$
f_{\mathrm{RKD}}(v)=n N_{\mathrm{RKD}}\left(1+\frac{v^{2}}{\kappa \Theta^{2}}\right)^{-\kappa-1} \exp \left(\frac{-\alpha^{2} v^{2}}{\Theta^{2}}\right) .
$$

The normalization constant is such that $\int \mathrm{d}^{3} v f_{\mathrm{RKD}}=n$ is fulfilled, and is given by

$$
N_{\mathrm{RKD}}=\frac{1}{(\pi \kappa)^{3 / 2} \Theta^{3}} U\left(\frac{3}{2}, \frac{3-2 \kappa}{2}, \alpha^{2} \kappa\right),
$$

where $U(a, c, x)$ denotes the Kummer function 25 (also known as Tricomi function). The RKD consists of a power-law term and a Maxwellian cutoff, which is independent of $\kappa$, but contains a regularization parameter $\alpha$, which needs to be positive but small enough in order to keep the main implication of the distribution and allow for convergent velocity moments and small contribution of superluminal particles. Despite its apparent ad-hoc application, there is also observational justification for the development of the RKD 2627

Figure 1 illustrates the impact of the regularization parameter $\alpha$ on the distribution function, where two RKDs ( $\kappa=2$;

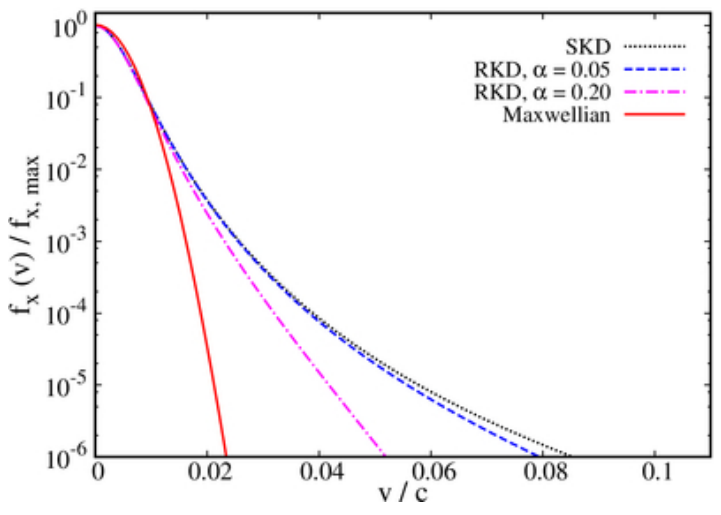

FIG. 1. Comparison of two RKDs $(\kappa=2, \alpha=0.05$, blue dashed line, and $\alpha=0.2$, pink dashed-dotted line), the Maxwellian $(\alpha=0$, $\kappa \rightarrow \infty$, solid red line), and the SKD $(\alpha=0, \kappa=2$, dotted black line). All distributions (normalized to their maximums) are plotted vs. particle speed $v_{\perp} \equiv v$ (normalized to the speed of light) at $v_{\|}=0$.

$\alpha=0.05$ and $\alpha=0.2)$ are compared with the corresponding SKD $(\alpha=0)$ and Maxwellian $(\alpha=0$ and $\kappa \rightarrow \infty)$. By applying the RKD, the contribution of superluminal particles can become negligible, if $\alpha$ is properly chosen. In Figure 1 we can see that more particles of higher speed are removed with increasing $\alpha$. On the other hand, the $\alpha$-parameter must be small enough, i.e., usually $\alpha \ll 1$, in order to keep a reasonable contribution of the suprathermals in the high-energy tails of the distribution.

In this paper we consider the (isotropic) RKD to study the electromagnetic (EM) stable modes, and for the unstable modes the (anisotropic) regularized bi-Kappa-distribution (RBKD), given by 21

$$
\begin{aligned}
f_{\mathrm{RBKD}}\left(v_{\|}, v_{\perp}\right)= & n N_{\mathrm{RBKD}}\left(1+\frac{v_{\|}^{2}}{\kappa \Theta_{\|}^{2}}+\frac{v_{\perp}^{2}}{\kappa \Theta_{\perp}^{2}}\right)^{-\kappa-1} \\
& \times \exp \left(-\frac{\alpha_{\|}^{2} v_{\|}^{2}}{\Theta_{\|}^{2}}-\frac{\alpha_{\perp}^{2} v_{\perp}^{2}}{\Theta_{\perp}^{2}}\right)
\end{aligned}
$$

and allowing for different regularization parameters $\alpha_{\|}$and $\alpha_{\perp}$ in directions parallel and perpendicular to the magnetic field, respectively. Here, the normalization constant reads 21

$$
N_{\mathrm{RBKD}}=\frac{1}{(\pi \kappa)^{3 / 2} \Theta_{\|} \Theta_{\perp}^{2} W},
$$

where the quantity $W$ is the integral

$$
W=\int_{0}^{1} \mathrm{~d} t U\left(\frac{3}{2}, \frac{3-2 \kappa}{2}, \kappa\left[\alpha_{\perp}^{2}+\left(\alpha_{\|}^{2}-\alpha_{\perp}^{2}\right) t^{2}\right]\right),
$$

which can be solved analytically if $\alpha_{\|}=\alpha_{\perp}$, and numerically otherwise. ${ }^{21}$

This paper is structured in the following way. In Section II we introduce the dispersion theory and present LEOPARD ${ }^{\mathrm{T}}$ (acronym for Linear Electromagnetic Oscillations in Plasmas 
with Arbitrary Rotationally-symmetric Distributions), the numerical solver used to solve the main dispersion relations. Section III contains the results, with detailed discussions of the stable modes, the electromagnetic electron-cyclotron and ion-cyclotron waves in Section III A, while Sections III B, III C and III D examine the instabilities of EM cyclotron and firehose modes. We conclude in Section IV with a summary of our present results and a discussion of the strengths of LEOP$A R D$, as well as the computational issues to be fixed in future works.

\section{GENERAL THEORY \& NUMERICAL SOLVER}

The hot and dilute plasmas from space are propitious to departures from thermal equilibrium and implicitly to various types of wave instabilities. These instabilities are described by solving the kinetic dispersion relation, which sets the (complex) wave frequency $\omega=\omega_{\mathrm{r}}+i \gamma$ and the wavenumber $k$ in relationship. From the beginning we need to mention that the analytical derivation of the dielectric tensor for a RKD, especially the anisotropic versions, is not straightforward. For this reason we proceed to test and use $L E O P A R D^{1}$, a numerical solver able to resolve the dispersion and stability properties of plasmas with arbitrary gyrotropic distribution functions defined numerically. This solver works with a general theoretical approach which may appeal any of the distribution models, including Maxwellians, SKDs and RKDs, to compute the dispersion curves for the wave frequency as well as growth or damping rate of the electromagnetic waves in a hot, homogeneous and magnetized plasma.

In the following we consider the plasma fluctuations to be sufficiently weak, so that linear theory can be applied to describe them. Typically, to derive the linear kinetic dispersion relation $\omega(k)$, one may start from the Vlasov-Maxwell equations and perform a Fourier and Laplace transformation of the perturbed quantities, which gives temporally and spatially variations for the EM fields and particle (flux) densities. These are inserted into Maxwell's equations to yield the general dispersion relation for nontrivial solutions 5 7/7

$$
0=\operatorname{det}\left(\frac{c^{2} k^{2}}{\omega^{2}}\left(\frac{\vec{k} \otimes \vec{k}}{k^{2}}-\mathbb{1}\right)+\overleftrightarrow{\varepsilon}(\vec{k}, \omega)\right)
$$

where $c$ is the speed of light, $k$ is the wavenumber, $\mathbb{1}$ is the unit tensor, and $\otimes$ is the dyadic product of two vectors. The dielectric tensor $\overleftrightarrow{\varepsilon}(\vec{k}, \omega)$ in Eq. (8) is defined as

$$
\overleftrightarrow{\varepsilon}(\vec{k}, \omega)=\mathbb{1}+\frac{i}{\omega \varepsilon_{0}} \overleftrightarrow{\sigma}(\vec{k}, \omega)
$$

with $\varepsilon_{0}$ being the permittivity of free space and $\overleftrightarrow{\sigma}(\vec{k}, \omega)$ the conductivity tensor. By linearizing the collision-free VlasovMaxwell equations the components of the dielectric tensor can be derived via 5128

$$
\begin{aligned}
\varepsilon_{i j}(\vec{k}, \omega)= & \delta_{i j}-2 \pi \sum_{\mathrm{s}} \frac{\omega_{\mathrm{ps}}^{2}}{\omega^{2}}\left\{\int_{-\infty}^{\infty} \mathrm{d} v_{\|}\right. \\
& \left.\times \int_{0}^{\infty} \mathrm{d} v_{\perp} v_{\perp}^{2} \sum_{n=-\infty}^{\infty} \frac{\omega}{\omega-n \Omega_{\mathrm{s}}-k_{\|} v_{\|}} Q_{i j}^{\mathrm{s}, n}\right\} .
\end{aligned}
$$

Here, $\delta_{i j}$ denotes the Kronecker delta, $\omega_{\mathrm{ps}}$ the plasma frequency, $\Omega_{\mathrm{s}}$ the cyclotron frequency of species $s$ and $n$ the Bessel function index. The elements $Q_{i j}^{\mathrm{s}, n}$ contain the derivatives of the particle velocity distribution function and are computed as described in Refs. 1 and 28. The only requirement for a distribution function $f_{\mathrm{s}}$ is gyrotropy, i.e., $\frac{\partial f_{\mathrm{s}}}{\partial \phi}=0$ with $\phi$ being the gyroangle, it is otherwise arbitrary. For the cases considered below we choose the isotropic RKD (Eq. 3) for the stable modes in Section III A and the anisotropic RBKD (Eq. 5) for the instabilities in Sections III B, III C and III D.

The code $\angle E O P A R D$ is a grid-based kinetic dispersion relation solver, which is written in Fortran-90 and is based on its predecessor DSHARK ${ }^{[29}$ The code can be applied to distributions of arbitrary shapes and is provided to public access 30 to be used and modified. LEOPARD solves the general linear dispersion relation (Eq. 8) by computing the dielectric tensor (Eq. 9) with its components in order to find complex solutions $\omega(k)$, i.e., the frequencies (i.e, real part $\mathfrak{R}(\omega) \equiv \omega_{\mathrm{r}}$ ) as well as the growth and damping rates (i.e., imaginary part $\mathfrak{I}(\omega) \equiv \gamma)$ of electromagnetic waves in homogeneous plasmas. The code allows for arbitrary wave propagation, i.e., parallel and oblique direction of wave propagation with respect to the background magnetic field, and for an arbitrary number of particle species. For the application of (bi-)Maxwellian velocity distributions a separately implemented routine can be used, which reduces the computation time significantly. However, other distributions have to be provided to the code as a $2 \mathrm{D}$ data grid in velocity space $\left(v_{\|}, v_{\perp}\right)$. In the code the distribution function is interpolated on the velocity grid with cubic splines, which approximate the function piecewise in a continuous and smooth way. Furthermore, the user specifies the wavenumber interval (normalized to the skin depth), its resolution and needs to provide the code with initial guesses for the real and imaginary part of $\omega$. While the code allows for an arbitrary number of particle species, the declaration of the first particle species is important, as all normalized quantities and values are given in units of that species.

\section{RESULTS}

The solutions of the general dispersion relation (Eq. 8) are derived numerically with the numerical solver LEOPARD, yielding wavenumber dispersion curves for the frequencies as well as the growth and damping rates of the considered parallel electromagnetic modes. We examine the effects of the regularization parameter $\alpha$ on the results and compare them to the ones obtained for the corresponding Maxwellian and standard Kappa-distributions, which serve as test cases 

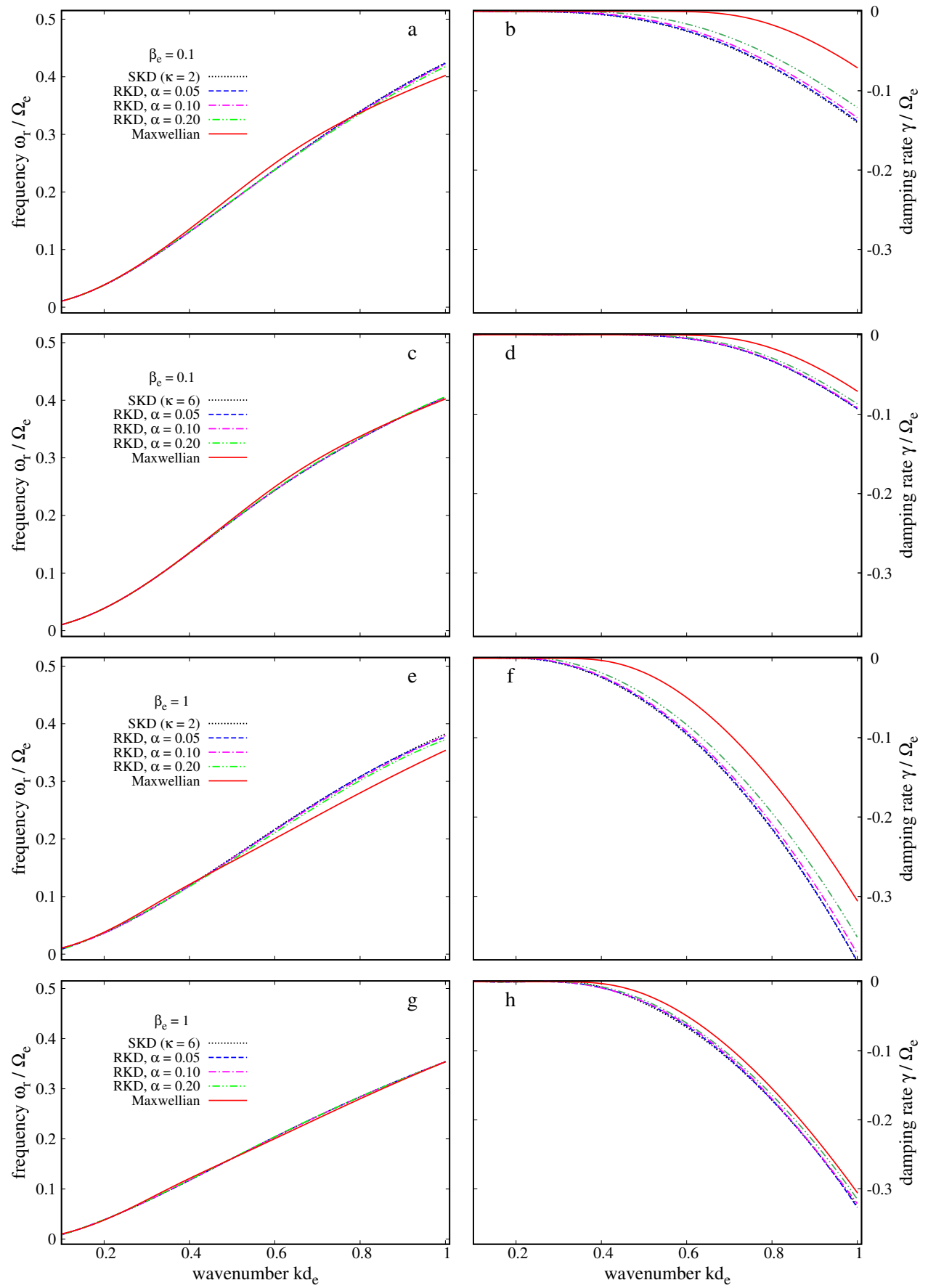

FIG. 2. Wavenumber dispersion for the EMEC frequency (left column) and damping rate (right column). Parameters are given in the legends.

in order to validate the functionality of the numerical code. While damped modes in Maxwellian-distributed plasmas are discussed widely, see the textbook of Gary, ${ }^{31}$ studies in general do not cover damped modes in Kappa-distributed plasmas. In the following we consider a two-component plasma consisting of protons $(\mathrm{s}=\mathrm{p})$ and electrons $(\mathrm{s}=\mathrm{e})$. In Section IIIA the isotropic RKD is applied and the dispersion curves and damping rates of stable electromagnetic electroncyclotron (EMEC) and ion-cyclotron (EMIC) modes are discussed. In Sections. III B and IIIC we analyze the insta- bilities of anisotropic electrons or protons, when described with RBKDs with $\alpha_{\|}=\alpha_{\perp}$. While Section IIIB presents the EMEC and EMIC instabilities, which are driven by a temperature anisotropy $A \equiv T_{\perp} / T_{\|}>1$, Section III C shows the results for the electron firehose $(\mathrm{EFH})$ and proton firehose $(\mathrm{PFH})$ instabilities, which occur for opposite anisotropies $A<1$. In Section IIID we revisit the EMEC, EMIC, EFH and PFH instabilities, and describe the anisotropic particle species with RBKDs with $\alpha_{\|} \neq \alpha_{\perp}$. 


\section{A. Damped Electromagnetic Cyclotron Modes}

The electromagnetic electron-cyclotron (EMEC) or whistler waves are often identified, directly or indirectly in observations of space plasmas, see Refs. 32 or 33 and references therein. EMEC waves are transverse electromagnetic modes with right-hand circular polarization and frequencies between the electron and ion gyrofrequencies, i.e., $\Omega_{\mathrm{i}}<\omega<\Omega_{\mathrm{e}}$, where $\Omega_{\mathrm{e}, \mathrm{i}}$ are absolute values of electron and ion (proton) gyrofrequencies, respectively $[34$ For the first time these modes were observed as whistlers in Earth's atmosphere,, 35 being enhanced through resonance with energetic electrons. The particles in a magnetized plasma are subject to gyromotion and cyclotron resonance with these modes, more exactly, with the transverse electric field of the parallel EM modes considered here. For particles that gyrate in phase with the electric field, the gyrofrequency $\Omega$ matches the Doppler (thermally) shifted wave frequency, and the cyclotron resonance condition reads 36

$$
v=\frac{\omega \pm \Omega}{k_{\|}} .
$$

The signs \pm are usually assigned to right-hand and left-hand polarized waves, respectively.

Figure 2 shows the frequencies (left panels) and damping rates (right panels) of stable EMEC modes for different values of $\kappa$ and $\beta_{\mathrm{e}}$ (for exact values see legends). In all plots the protons are assumed to be Maxwellian $\left(\beta_{\mathrm{p}}=1\right)$, where the electrons are described by RKDs of different values of $\alpha$. As test cases ${ }^{31}$ the results of the Maxwellian (RKD with $\alpha=0$ and $\kappa \rightarrow \infty$ ) are computed and shown in each plot. The results for the SKD (with $\alpha=0$ ) are also displayed as references to be compared with the RKD. A comparison to the corresponding SKD reveals that the wave frequency dispersion curves remain basically unaffected even for higher values of the $\alpha$ parameter. We quantify the difference in the damping rates (or growth rates discussed in Secs. III B, III D obtained for RKDs by comparison to the corresponding SKD, by the following deviation

$$
R=\left|1-\frac{\gamma_{\max , \mathrm{RKD}}}{\gamma_{\max , \mathrm{SKD}}}\right| .
$$

For the damping rates of the EMEC modes we estimate a $13 \%$ deviation at a given value $k d_{\mathrm{e}}=1$, and for $\kappa=2, \beta_{\mathrm{e}}=0.1$ and $\alpha=0.2$. For the lower $\alpha$-values this deviation decreases to about $5 \%$. The results for $\beta_{\mathrm{e}}=1$ show for all values of $\alpha$ a deviation below 9\%. All deviations are presented in Tab. I. For $\kappa=6$ the deviations in the damping rates are overall below $8 \%$. Differences may increase with increasing wavenumber, where the mode is highly damped, i.e., shifted to lower wave frequencies and lower damping rates with an increase of $\alpha$. For $\kappa=6$ the differences are much less significant as the distribution models are closer to Maxwellian limits. Damping rates for the SKD plasma remain below the Maxwellian, and for the RKD it shifts toward the Maxwellian with increasing $\alpha$. This trend occurs due to the increasing value of $\alpha$, by which more resonant particles that can gain energy from the

\begin{tabular}{|c|c|c|c|c|c|c|c|c|c|c|c|}
\hline \multicolumn{3}{|c|}{ EMEC (0.1) } & \multicolumn{3}{|c|}{ EMEC (1) } & \multicolumn{3}{|c|}{$\operatorname{EMIC~(0.1)~}$} & \multicolumn{3}{|c|}{ EMIC(1) } \\
\hline & $\alpha$ & $R$ & $\kappa$ & $\alpha$ & $R$ & $\kappa$ & $\alpha$ & $R$ & $\kappa$ & $\alpha$ & $R$ \\
\hline & 0.05 & 0.01 & 2 & 0.05 & $<1 \%$ & 2 & 0.05 & $<1 \%$ & 2 & 0.05 & $<1 \%$ \\
\hline & 0.1 & 0.04 & 2 & 0.1 & 0.02 & 2 & 0.1 & 0.02 & 2 & 0.1 & 0.02 \\
\hline 2 & 0.2 & 0.13 & 2 & 0.2 & 0.08 & 2 & 0.2 & 0.08 & 2 & 0.2 & 0.07 \\
\hline 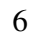 & 0.05 & $<1 \%$ & 6 & 0.05 & $<1 \%$ & 6 & 0.05 & $<1 \%$ & & 0.05 & $<1 \%$ \\
\hline & 0.1 & 0.02 & 6 & 0.1 & 0.01 & 6 & 0.1 & 0.01 & & 0.1 & 0.01 \\
\hline 6 & 0.2 & 0.07 & 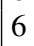 & 0.2 & 0.03 & 6 & 0.2 & 0.04 & & 0.2 & 0.02 \\
\hline
\end{tabular}
electromagnetic waves, are removed (see, e.g., Ref. 37).
TABLE I. The deviations for the damping rates of EMEC (at $\left.k d_{\mathrm{e}}=1\right)$ and EMIC (at $k d_{\mathrm{p}}=1.4$ ) modes, as provided by Eq. [12. The numbers in the brackets denote $\beta$.

The electromagnetic ion-cyclotron modes (EMIC) are low-frequency modes, left-hand circularly polarized and have been detected in the solar wind 38 and in planetary magnetospheres. ${ }^{39}$ In Fig. 3 the computed frequencies and damping rates are presented for stable EMIC modes. The setup and organization of the panels is the same as for Fig. 2 , except that the protons are described by RKDs now and electrons are Maxwellian and isotropic with $\beta_{\mathrm{e}}=1$. We can see that the wave frequency curves for the SKD and RKDs reach a plateau or maximum around $k d_{\mathrm{p}} \gtrsim 0.8$, while the same curves in the Maxwellian-distributed plasmas continue to increase ${ }^{31}$. At higher temperatures the wave frequency decreases with increasing the wavenumber. The effect is more significant for low values of the $\kappa$-index, i.e., $\kappa=2$, since with the increase of $\kappa$ the distributions approach the Maxwellian (and with the increase of $\alpha$ the dispersion curves overall approach the Maxwellian). Depending on the kinetic temperature of SKD particles, for low values of $\kappa$ one may critically need to invoke RKD models, which reduce the unrealistic contribution of superluminal particles present in the SKD models, see the $\kappa=2$ case in Ref. 23. The influence on the damping rates is similar to the EMEC modes: comparing to Maxwellian limits, in Kappa-distributed plasmas the waves are stronger damped, but with the increase of the $\alpha$-parameter more resonant particles are removed and the damping curves approach the Maxwellian. The deviation of the RKD-based damping rates from the one based on the SKD is overall below 9\%. All deviations are calculated at $k d_{\mathrm{p}}=1.4$ and displayed in Tab.I.

\section{B. Electromagnetic Cyclotron Instabilities}

In space plasmas, the free energy of anisotropic electron populations, e.g., an electron temperature anisotropy of $A_{\mathrm{e}}=$ $T_{\mathrm{e} \perp} / T_{\mathrm{e} \|}>1$, may lead to an instability of the EMEC (or whistler-cyclotron) modes, with maximum growth rates in parallel direction. $\mathrm{By}$ convention, the growth is described via a positive rate $\gamma>0$. In the low-collisional plasmas from space, the enhanced whistler fluctuations selfconsistently induced by the electron anisotropy can be very effective in constraining large deviations from isotropy, and the observations appear to confirm that ${ }^{32}$ The frequency curves (left panels) and growth rates (right panels) of the EMEC instability computed for an RBKD-plasma are displayed in Fig. 4. Each plot 

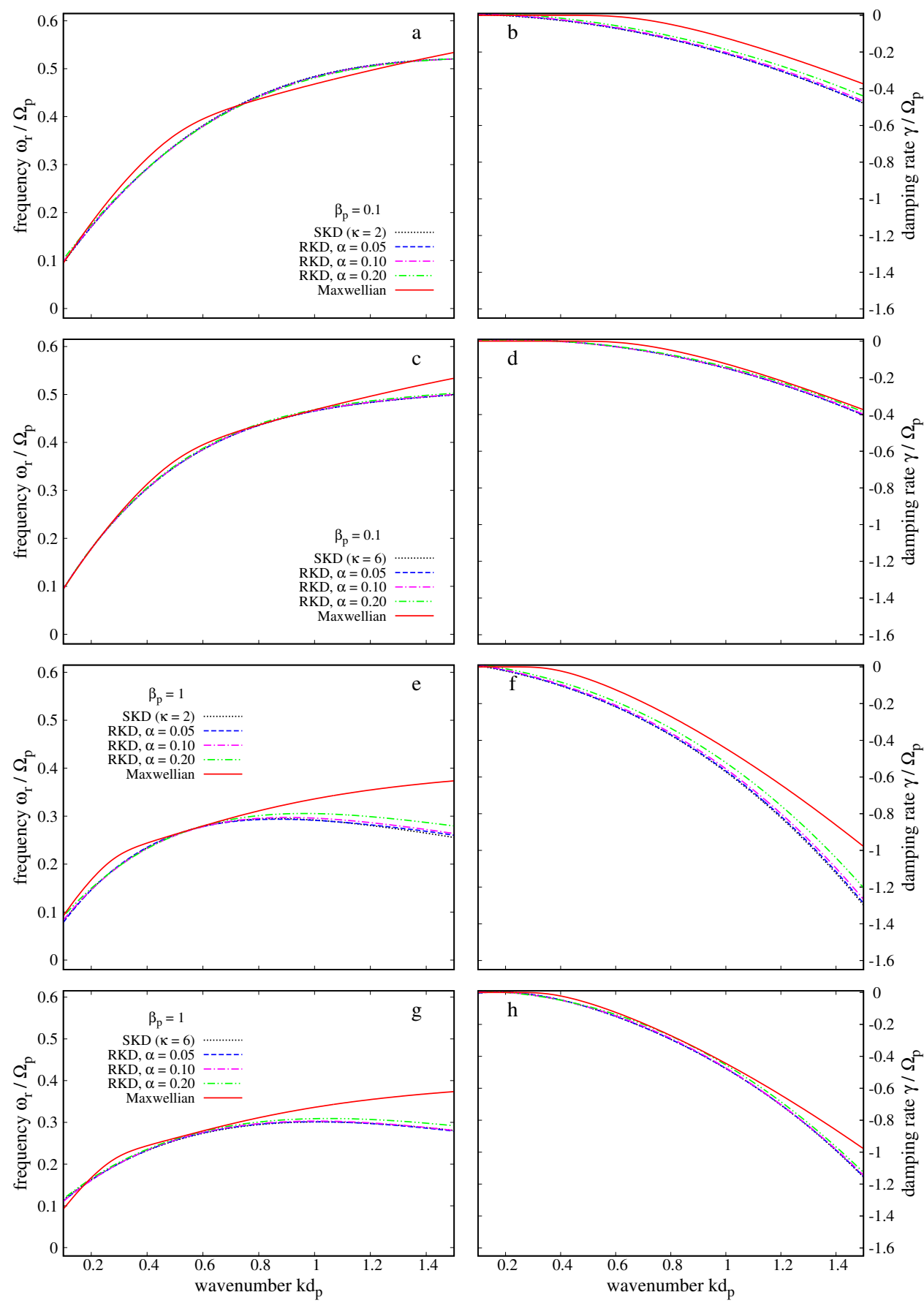

FIG. 3. Wavenumber dispersion for the EMIC frequency (left column) and damping rate (right column). Parameters are given in the legends.

contains the results for three RBKDs, which are compared to the corresponding SBKD and Bi-Maxwellian distribution. ${ }^{6}$ The exact values of $\kappa, \beta_{\mathrm{e} \perp}$ and the anisotropy are displayed in the legends. The protons are assumed to be isotropic and Maxwellian with $\beta_{\mathrm{p}}=1$. In the left column of Fig. 4 the wave frequency dispersion curves remain basically unchanged for small anisotropies, e.g., $A_{\mathrm{e}}=1.5$, and with the increase of the anisotropy, e.g., for $A_{\mathrm{e}}=3$, all Kappa-curves deviate stronger from the Maxwellian. This difference is reduced for higher spectral indices, e.g., for $\kappa=4$, and the influence of the cutoff parameter $\alpha$ is minor. By comparing the maximums of the growth rates obtained for the RBKD with the one obtained for the SBKD we find the greatest deviation with $16 \%$ for $\kappa=2$, $A=1,5$ and $\alpha=0.2$, while the other curves mostly deviate to about 1 to $5 \%$ in their maximums. All deviations are presented in Tab. III The growth rates, displayed in the right column, are in general highly modified under the influence of the $\kappa$ - and $\alpha$-parameters. Profiles are the same for all cases, showing peaking (maximum) values increasing with the increase of the anisotropy that drives the instability. With the increase of $\alpha$ 

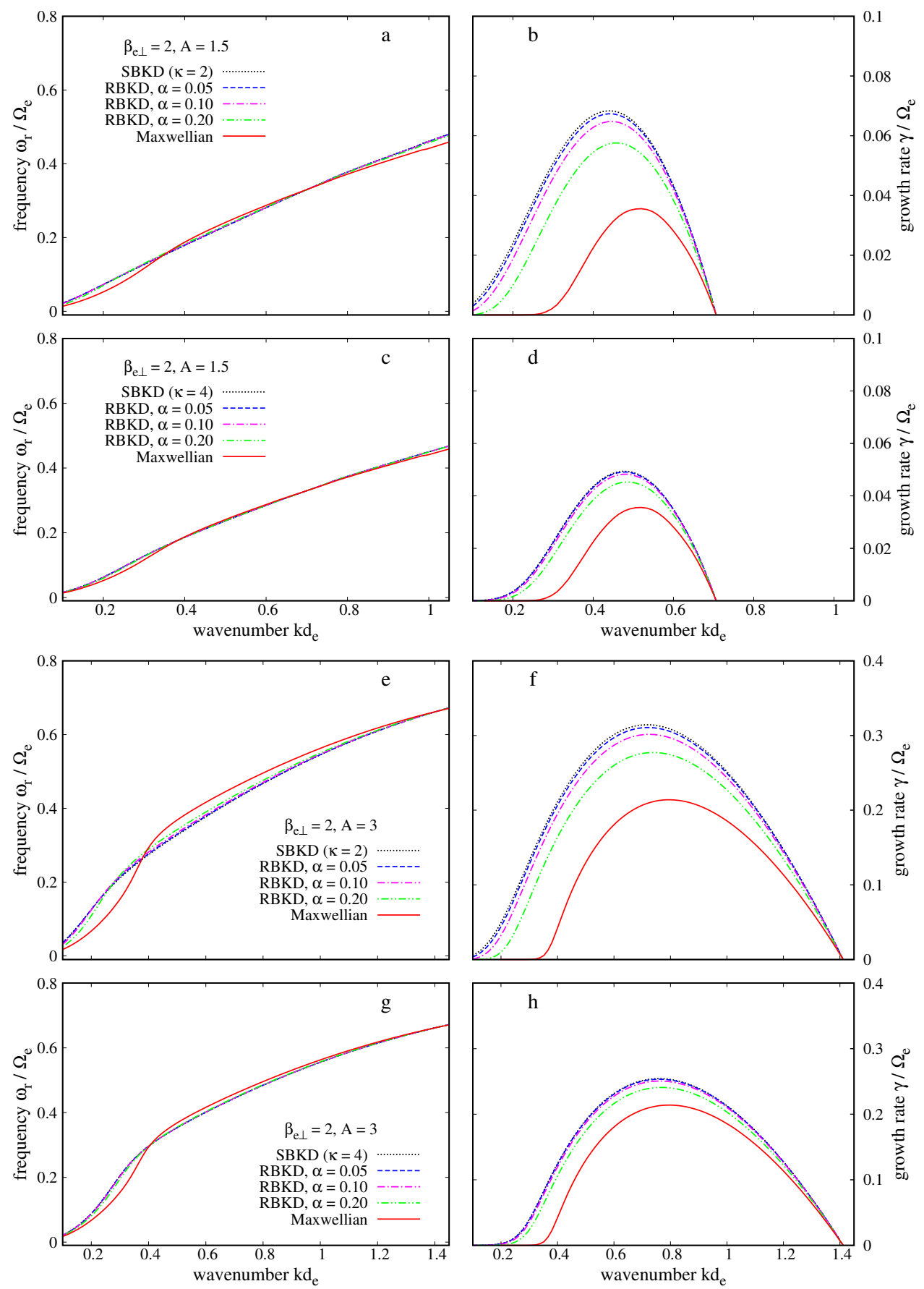

FIG. 4. Dispersion curves for wave frequencies (left column) and growth rates (right column) of the EMEC instability with $\alpha_{\|}=\alpha_{\perp}$. Parameters are explained in the legends.

the growth rates decrease with the peak shifting toward higher wavenumbers, and this effect can be understood in the following way. With increasing $\alpha$ the distribution function receives a greater cutoff in the high-velocity range, and the number of particles that resonate with, and lose energy to the waves is diminished. Consequently, the growth rates are reduced. Again, the influence of $\alpha$ on the growth rates is reduced for a higher $\kappa$.

We should mention that for the cyclotron resonant instabili- ties not only the number of resonant particles is responsible to determine the magnitude of the growth rate, but also the pitch angle anistropy of the velocity distribution in the area of resonance in the distribution that has to be sufficiently anisotropic, see Ref. 41 and 42 .

The EMIC instability arises due to an excess of kinetic energy of the protons in perpendicular direction, i.e., $T_{\mathrm{p} \perp}>T_{\mathrm{p} \|}$. The maximum growth rates of the EMIC waves are again obtained for parallel propagation, so that one can take $k \simeq k_{\|}$. 

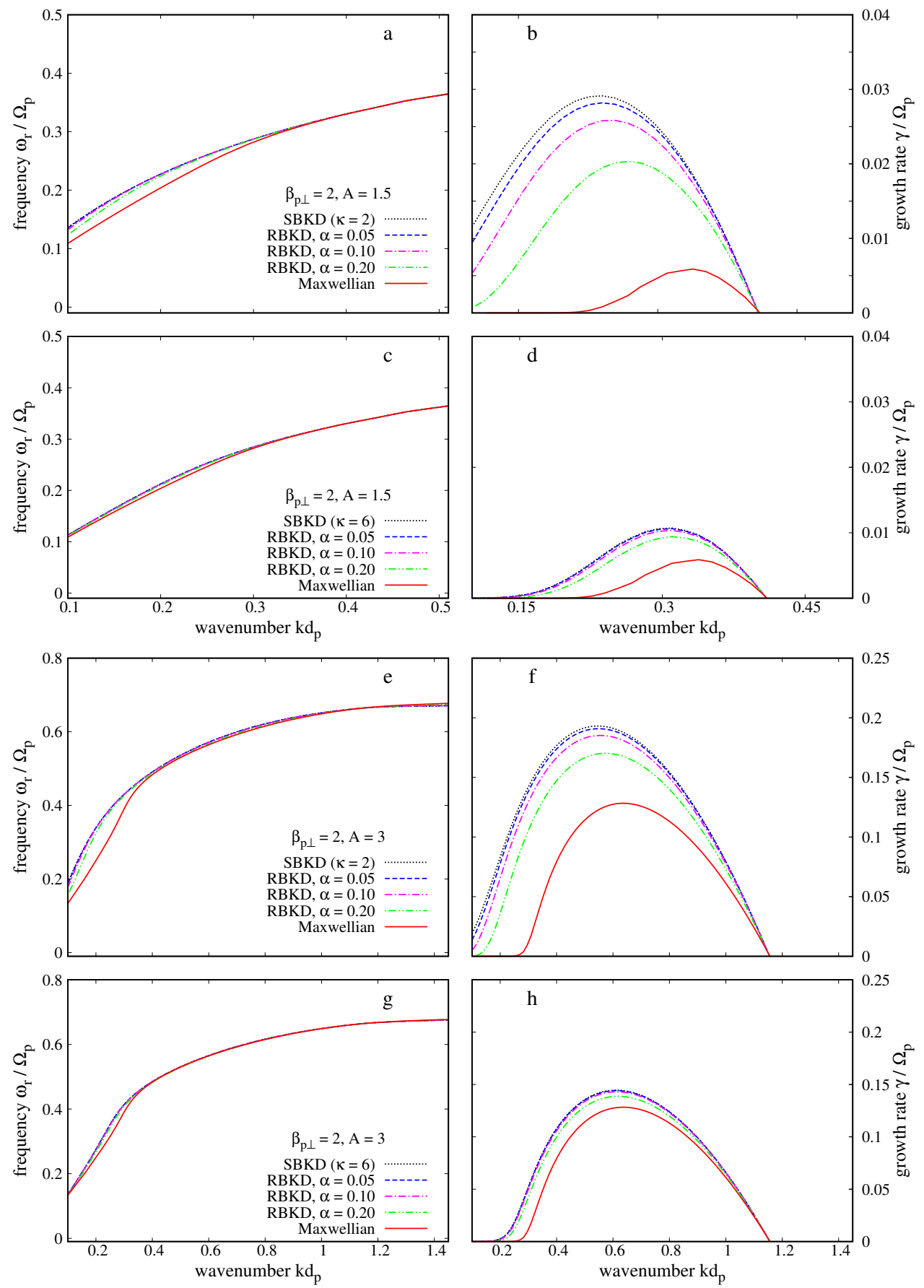

FIG. 5. Dispersion curves for wave frequencies (left column) and growth rates (right column) of the EMIC instability with $\alpha_{\|}=\alpha_{\perp}$. Parameters are explained in the legends.

Figure 5 shows the wave frequency curves and growth rates. The composition of the plots is similar as for the EMEC instability, where we have computed as test cases the results for the SBKD ${ }^{43}$ and the Bi-Maxwelliari ${ }^{43}$, assuming this time $\beta_{\mathrm{e}}=1$, while the protons are described by RBKDs. For the details of the parameter setup see the legends. While the dispersion curves are only slightly affected again by the change in the $\alpha$-parameter, the growth rates decrease with the increase in $\alpha$ (and hence a removal of resonant particles that lose energy to the waves) and the peak of the growth rates shifts toward higher wavenumbers. For $\kappa=6$ these effects are less prominent. When comparing the maximum growth rates we find for the EMIC instability for $\kappa=2, A=1,5$ and $\alpha=0.2$ the greatest deviation with about $30 \%$, while all other results show a deviation around $10 \%$ for the curves with $\alpha=0.2$, and mostly below 5\% for the curves with lower values of $\alpha$. All results are displayed in $\mathrm{Tab}$. 

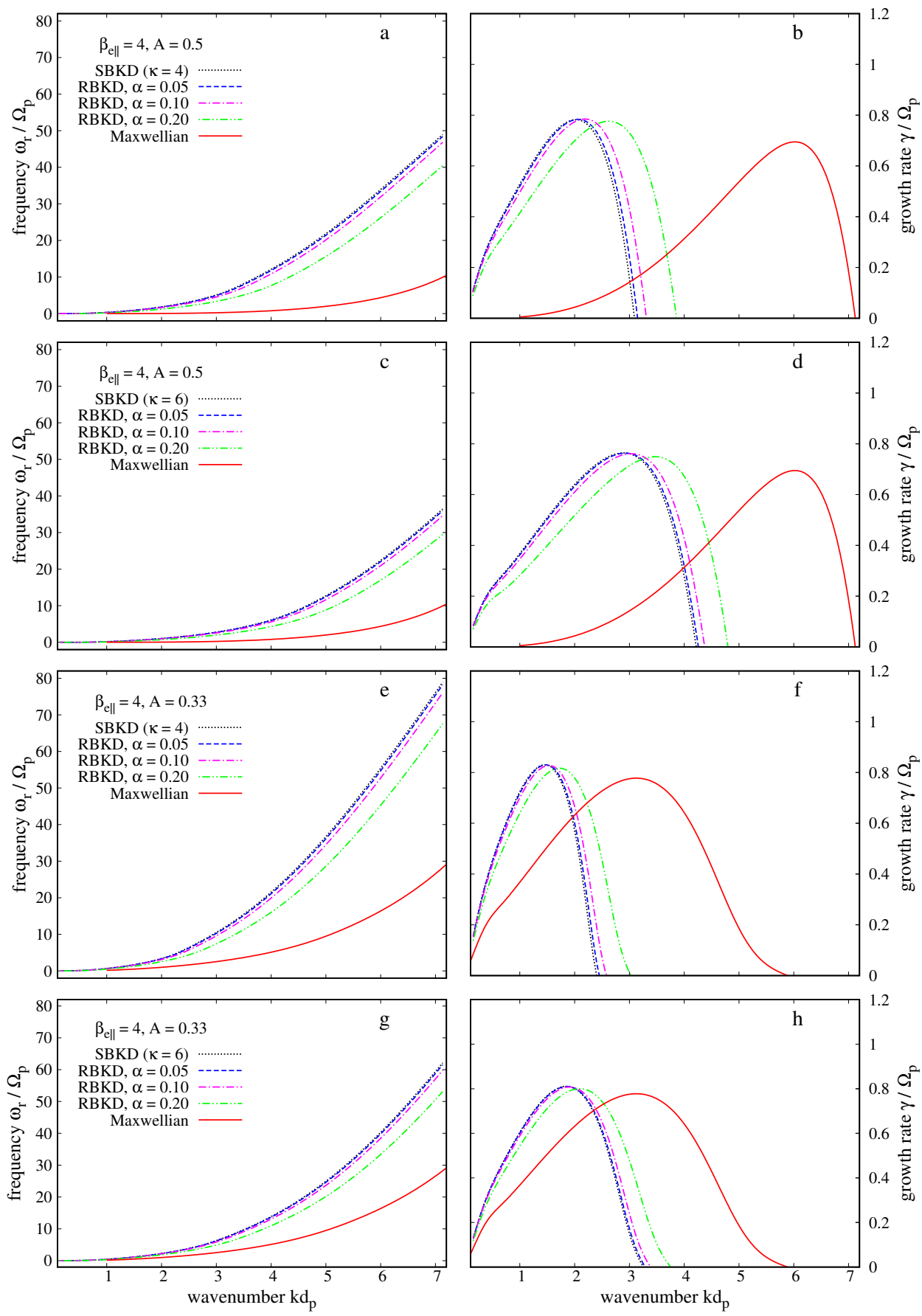

FIG. 6. Dispersion curves for wave frequencies (left column) and growth rates (right column) of the EFH instability with $\alpha_{\|}=\alpha_{\perp}$. Parameters are explained in the legends.

\section{The Electron and Proton Firehose Instabilities}

For opposite anisotropies of electrons, i.e., $A_{\mathrm{e}}<1$, the theory predicts the so-called electron firehose (EFH) instability, developing as a low-frequency left-hand circularly polarized mode in parallel direction, at wavenumbers $k d_{\mathrm{e}}>1$ and with frequencies between the proton cyclotron frequency and the electron cyclotron frequency $\left.\left(\Omega_{\mathrm{p}}<\omega_{\mathrm{r}}<\Omega_{\mathrm{e}}\right)\right)^{\frac{31}{31}}$ The parallel firehose instability is competed by another aperiodic branch propagating only obliquely to the magnetic field, see Refs. 44 and 45 and references therein, but our present study intended to testing regularized Kappa-distributions is limited to parallel EM modes.

Figure 6 shows the wave frequency curves (left column) and growth rates (right column) of the EFH instability. The legends summarize the values used for the model parameters and the protons are described by an isotropic Maxwellian of $\beta_{\mathrm{p}}=1$. Profiles of the wavenumber dispersion curves remain 

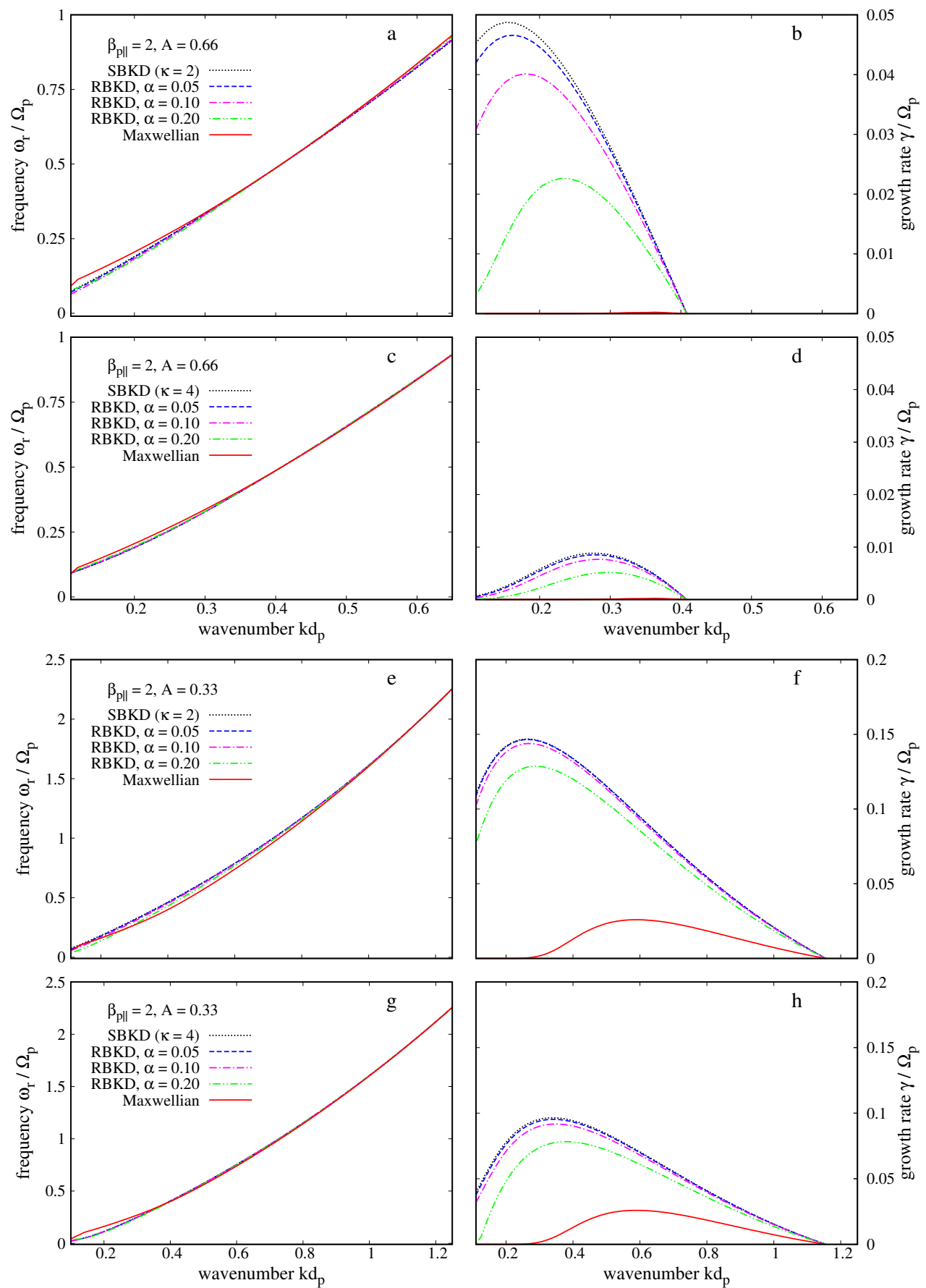

FIG. 7. Dispersion curves for wave frequencies (left column) and growth rates (right column) of the PFH instability with $\alpha_{\|}=\alpha_{\perp}$. Parameters are explained in the legends.

unchanged, as already known for the standard bi-Maxwellian and SBKD ${ }^{40}$, i.e., with the decrease of the temperature anisotropy the frequencies are significantly enhanced, while the peak of the growth rates increases and shifts toward lower wavenumbers. Decreasing $\kappa$ has similar effect, but with increasing $\alpha$ the growth rates shift toward higher wavenumbers towards the Maxwellian-based curves. The comparison of the maximum growth rates shows beside the shift of the peak almost no deviation between the RBKD- and SBKD-based re- sults, where the deviation is for almost all results below $1 \%$ (see, Tab. II for detailed results).

The proton firehose (PFH) instabilities, either parallel or the aperiodic oblique branches, arise when protons are characterized by a temperature anisotropy $A_{\mathrm{p}}<1$ and a sufficiently high $\beta_{\mathrm{p} \|}>1 \stackrel{41}{ }$ Different observations suggest that these firehose instabilities limit any further increase of the proton temperature in parallel direction. If the temperature anisotropy exceeds the instability threshold, the fluctuations excited by the 

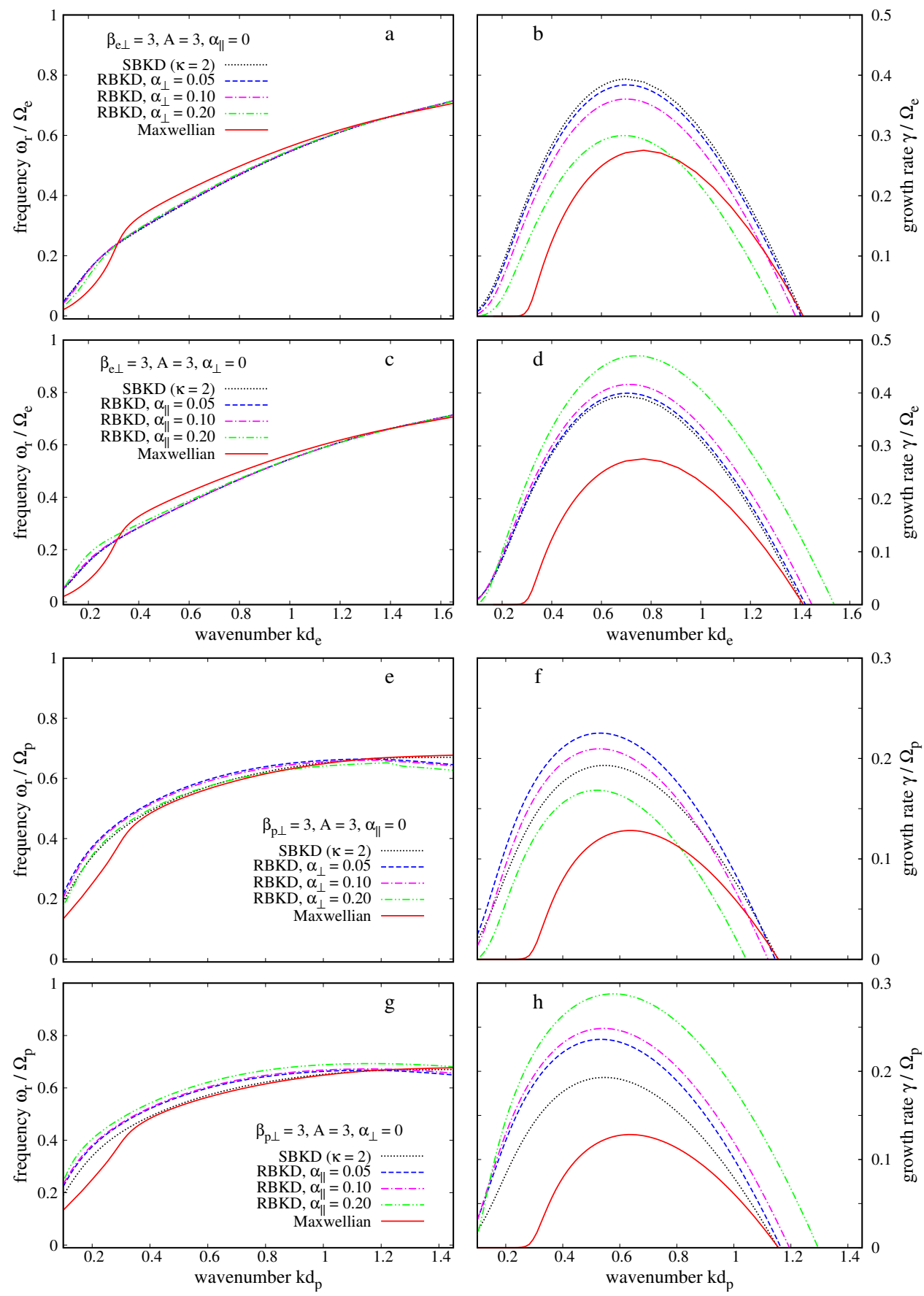

FIG. 8. Dispersion curves for wave frequencies (left panels) and growth rates (right panels) of the EMEC (top two rows) and EMIC (bottom two rows) instability with $\alpha_{\|} \neq \alpha_{\perp}$. Parameters are explained in the legends.

firehose modes grow and act back on the protons contributing to their relaxation and constraining their anisotropy ${ }^{[46}$ For certain conditions maximum growth may occur at parallel propagation, i.e., $\vec{k} \times \vec{B}_{0}=\overrightarrow{0}$, with $\vec{B}_{0}$ being the vector of the local background magnetic field, motivating our limitation to parallel modes in the present analysis. The proton firehose instability shows right-hand circular polarization in parallel direction of propagation and evolves out of the magnetosonic/whistler wave as the proton temperature anisotropy increases. $\frac{31}{\text { Fig- }}$ ure 7 presents the dispersion curves for the wave frequencies (left column) and growth rates (right column) of the PFH instability computed for the RBKD-distributed plasmas with different values of $\alpha$, together with the test case results for the SBKD- and Maxwellian distributed plasmas. All results are shown for two different values of $\kappa$ and $A_{\mathrm{p}}$, while the electrons are now described by an isotropic Maxwellian of $\beta_{\mathrm{e}}=1$. As in previous studies ${ }^{46}$ comparing SBKD with Maxwellian, the frequency changes with $A_{\mathrm{p}}$, but does not vary much with $\kappa$. It 

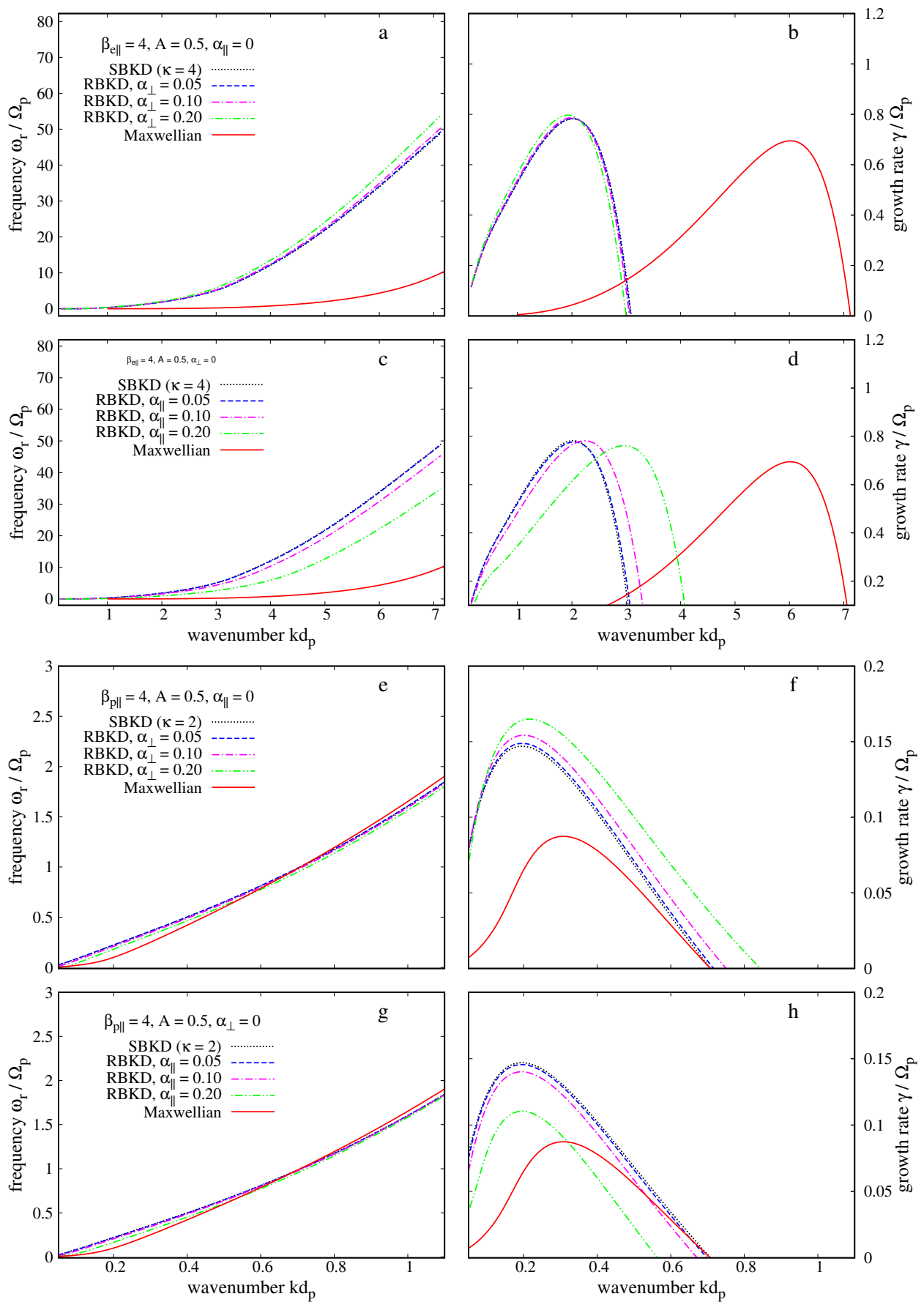

FIG. 9. Dispersion curves for wave frequencies (left panels) and growth rates (right panels) of the EFH (top two rows) and PFH (bottom two rows) instability with $\alpha_{\|} \neq \alpha_{\perp}$. Parameters are explained in the legends.

remains also unaffected by the variation of the $\alpha$-parameter. However, the growth rates and their peaking values are in general much higher than Maxwellian limit. For small $A_{\mathrm{p}}$ and small $\kappa$, e.g., Fig 7 panel b with $\kappa=2$ and $A_{\mathrm{p}}=0.66$, the growth rates decrease significantly (the maximum decreases by about 53\%) with increasing $\alpha$ and shift toward higher wavenumbers. Another rather significant decrease by $41 \%$ in the maximun growth rate occurs in panel d with $\kappa=4$ and $A_{\mathrm{p}}=0.66$, while the differences for all other curves are be- low $20 \%$ (see, Tab. II]) For higher values of $\kappa$, e.g., panel d, or higher anisotropies, e.g, panel f, these effects become less prominent.

\section{Anisotropic regularization parameters}

In Sections. III B and $\amalg$ II C we examined the effects of an isotropic regularization using the same values for parameter 


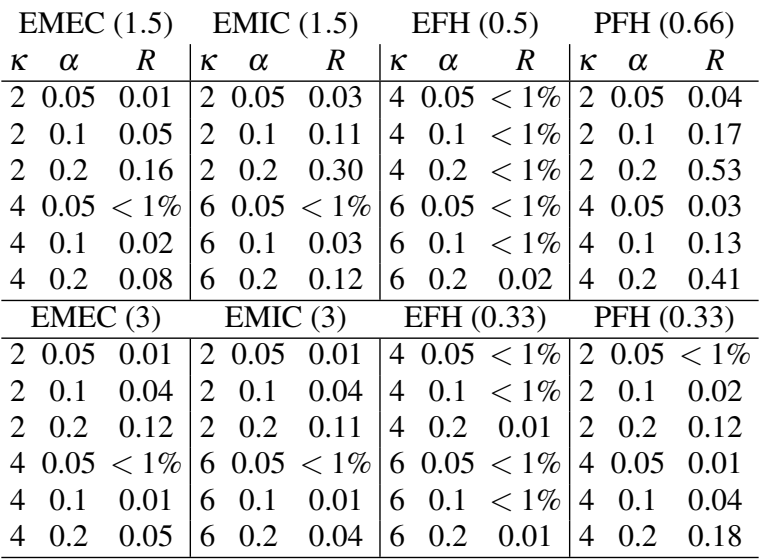

TABLE II. The deviations for the maximum growth rates of the EMEC, EMIC, EFH and PFH instabilities, as provided by Eq. (12). The numbers in the brackets denote the anisotropy $A$.

$\alpha$, i.e., $\alpha_{\|}=\alpha_{\perp}$, in both parallel and perpendicular directions. As for a more general case in this section we allow for $\alpha$ to be anisotropic, i.e., $\alpha_{\|} \neq \alpha_{\perp}$ and show the effects of this regularization on the wavenumber dispersion properties of the EMEC, EMIC, EFH and PFH instabilities.

Figure 8 displays the results for the EMEC (top two rows) and EMIC (bottom two rows) instabilities, where we examine the effect of changing the regularization parameter only in one direction, while keeping it constant in the other direction (see the legends). The plots for the EMEC instability show the frequencies (left column) and growth rates (right column) for the case of a constant $\alpha_{\|}$and varied $\alpha_{\perp}$ (panels a and b) and the case of a constant $\alpha_{\perp}$ and different values of $\alpha_{\|}$(panels $\mathrm{c}$ and d). We assume $\kappa=2, \beta_{\mathrm{e} \perp}=3, \beta_{\mathrm{p}}=1$ and $A_{\mathrm{e}}=3$ for all plots. Although similar to the previous case with $\alpha_{\|}=\alpha_{\perp}$ in Fig. 4 , the wave frequency is slightly lowered with increasing $\alpha_{\perp}$ or slightly enhanced with increasing $\alpha_{\|}$. On the other hand, the growth rates are significantly affected by the change of the regularization parameters. In the case of constant $\alpha_{\|}$(panel b), the growth rates markedly decrease with the increase of $\alpha_{\perp}$ (peak decreases by $23 \%$ compared to the SBKD-based result), and the peak shifts toward lower wavenumbers. All differences in the maximums of the growth rates between RBKDand SBKD-based results are shown in TabIII Physically, this can be explained by the variations of the effective anisotropy, which drives the EMEC instability. In the case of $\alpha_{\|} \neq \alpha_{\perp}$ the anisotropy becomes a function of the two regularization parameters and thus a change in either $\alpha_{\|}$or $\alpha_{\perp}$ alters the anistropy. With the increase of $\alpha_{\perp}$ (and a constant $\alpha_{\|}$), the distribution loses more high-energy particles from perpendicular direction that can resonate with the waves, and the effective anisotropy decreases, which is reflected by lower peaks of the growth rates. However, the same effective anisotropy increases if $\alpha_{\perp}$ is constant but $\alpha_{\|}$increases, leading in this case to a stimulation of the instability. For the EMIC instability we find a similar behavior of the results that can be explained analogously, i.e., the dispersion curves for the wave frequency remain basically unchanged with increasing com- ponents of the $\alpha$-parameter, while the growth rates change in the way described for the EMEC instability. Rather big differences occur in panel $\mathrm{h}$, where the maximum of the curve with $\alpha_{\|}=0.05$ deviates by $22 \%$ from the SBKD-curve, $\alpha_{\|}=0.1$ by $28 \%$, and $\alpha_{\|}=0.2$ by almost $50 \%$. Detailed results for the EMIC instability are shown in Tab. III.

\begin{tabular}{ccc|ccc|ccc|ccc}
\multicolumn{3}{c}{ EMEC } & \multicolumn{3}{c}{ EMIC } & \multicolumn{3}{c}{ EFH } & \multicolumn{3}{c}{ PFH } \\
$\alpha_{\|}$ & $\alpha_{\perp}$ & $R$ & $\alpha_{\|}$ & $\alpha_{\perp}$ & $R$ & $\alpha_{\|}$ & $\alpha_{\perp}$ & $R$ & $\alpha_{\|}$ & $\alpha_{\perp}$ & $R$ \\
\hline 0 & 0.05 & 0.02 & 0 & 0.05 & 0.16 & 0 & 0.05 & $<1 \%$ & 0 & 0.05 & 0.01 \\
0 & 0.1 & 0.08 & 0 & 0.1 & 0.08 & 0 & 0.1 & $<1 \%$ & 0 & 0.1 & 0.05 \\
0 & 0.2 & 0.23 & 0 & 0.2 & 0.12 & 0 & 0.2 & 0.02 & 0 & 0.2 & 0.12 \\
0.05 & 0 & 0.01 & 0.05 & 0 & 0.22 & 0.05 & 0 & $<1 \%$ & 0.05 & 0 & 0.01 \\
0.1 & 0 & 0.05 & 0.1 & 0 & 0.28 & 0.1 & 0 & $<1 \%$ & 0.1 & 0 & 0.04 \\
0.2 & 0 & 0.19 & 0.2 & 0 & 0.49 & 0.2 & 0 & 0.02 & 0.2 & 0 & 0.24 \\
\hline
\end{tabular}

TABLE III. The deviations for the maximum growth rates of the EMEC and EMIC $(A=3, \kappa=2)$, EFH $(A=0.5, \kappa=4)$ and PFH $(A=0.5, \kappa=2)$ instabilities, as provided by Eq. (12).

In Fig. 9 we show the effect of different $\alpha_{\|}$and $\alpha_{\perp}$ on the EFH (top two rows) and the PFH (bottom two rows) instabilities. Again, we can see that both the frequency (left panels) and growth rates (right panels) vary with the increase of the $\alpha$-components, especially for the EFH instability. However, comparing to the EMEC and EMIC instabilities, here we observe opposite effects, as for instance, for the EFH instability the frequency is lowered with increasing $\alpha_{\perp}$, but markedly lowered with increasing $\alpha_{\|}$. In this case the growth rates are not much affected by $\alpha_{\perp}$ (i.e., maximum growth rates deviate by less than $3 \%$ ), but are slightly lowered moving to higher wavenumbers for higher values of $\alpha_{\|}$. The PFH wave frequencies do not change much, but the growth rates increase with increasing $\alpha_{\perp}$ and decrease with increasing $\alpha_{\|}$. The largest deviation occurs for a strong cut-off parameter $\alpha_{\|}=0.24$ in panel $\mathrm{h}$, where the maximum growth rate deviates by $24 \%$.

\section{SUMMARY AND OUTLOOK}

There is consistent evidence that shows the existence of particle populations in space plasmas, e.g., in the solar wind and planetary magnetospheres, to be out of thermal equilibrium and showing enhanced suprathermal tails that can be wellfitted by standard Kappa-distributions (SKD). Due to unphysical limitations of the SKD, the regularized Kappa-distribution (RKD) has been introduced, which removes the critical restrictions of the SKD and is able to reproduce as limit cases the Maxwellian and the SKD as well as its results considering plasma waves and instabilities. We have investigated for the first time parallel electromagnetic modes in a plasma described by regularized Kappa-distributions and used parameters typical for space plasmas, but not restrained to these conditions. The results were derived by using the kinetic dispersion solver LEOPARD able to resolve the dispersion properties for plasma particles with arbitrary distribution functions. The wavenumber dispersion curves obtained for Maxwellian and SKD distribution functions are in perfect agreement with 
those predicted by the numerical solvers dedicated to these models.

In Section III A we presented the dispersion curves for wave frequencies and damping rates derived with $L E O P A R D$ for the stable electromagnetic electron-cyclotron (EMEC) and ioncyclotron (EMIC) modes by applying the isotropic RKD. The results show that even for higher values of the regularization parameter $\alpha$ the dispersion curves show almost no deviation from the curves obtained for the SKD. However, for the damping rates a value of $\alpha<0.1$ is necessary to reproduce the data of the SKD, i.e., allowing only for small deviations from the SKD-based damping rates.

In Section III B we used the anisotropic regularized Kappadistribution (RBKD) and computed the frequencies and growth rates of the EMEC and EMIC instabilities, which are driven by a temperature anisotropy of $A=T_{\perp} / T_{\|}>1$. Here, we could also see that the frequencies even for relative high values of $\alpha_{\|}=\alpha_{\perp}=\alpha$ are close to the results of the corresponding anisotropic standard Kappa-distribution (SBKD) and that the growth rates decrease with increasing $\alpha$ (as with higher values of $\alpha$ there are less resonant particles to drive the instability). For the electron firehose (EFH) and proton firehose (PFH) instabilities in Section III C we found similar results, except the increase of the growth rates for increasing $\alpha_{\perp}$ (while keeping $\alpha_{\|}$constant), as the effective temperature anisotropy increases in that case. For anisotropic regularization parameters, i.e., $\alpha_{\|} \neq \alpha_{\perp}$, examined in Section III D the growth rates of the EMEC and EMIC instabilities decrease with increasing $\alpha_{\perp}$ (keeping $\alpha_{\|}$constant), and increase with increasing $\alpha_{\|}$(keeping $\alpha_{\perp}$ constant), which is due either to a decrease of the effective (temperature) anisotropy for the former case, or a higher anisotropy in the latter case. For the $\mathrm{EFH}$ and PFH instabilities we found the opposite case, i.e., an increase of the growth rates with increasing $\alpha_{\perp}$ and a decrease with increasing $\alpha_{\|}$(keeping the component in the other direction constant, respectively).

The regularized Kappa-distribution, therefore, can reproduce the results of a standard Kappa-distribution with the benefit of removing its limitations by being defined for all $\kappa>0$, having no diverging velocity moments and no contribution to macroscopic quantities (e.g., pressure) by superluminal particles (provided that $\alpha$ is choosen properly). As a continuation of the wave dispersion properties of plasma systems described by the regularized Kappa-distribution, future studies must consider oblique modes, $\frac{47}{\mathrm{e}} \mathrm{eg}$., the aperiodic mirror ${ }^{48}$ and firehose $45[49 \mid 50$ instabilities. However, along with these strengths, we have encountered a few issues which need only to be mentioned at this stage. The code is very effective in the study of kinetic instabilities, such as the EMEC, EMIC and PFH instabilities with the assumption of a two-component plasma (electrons and protons) with only one anisotropic component, while the other component is assumed to be isotropic and Maxwellian. Since the code has the option to produce the Maxwellian distribution internally, this reduces the computation time significantly. Once the proper initial guesses for the real and imaginary part of $\omega$ were found, the code worked solidly for these cases. However, the choice of the initial guesses is crucial for the code to start on the desired solution branch. Difficulties occurred when we considered damped modes, i.e., when applied isotropic distributions for all particle species. The results in Figs. 2 and 3 had to be smoothed by an interpolation method, because the raw results from $L E O P$ $A R D$ showed many irregularities toward higher wavenumbers in the trend of the curves. The reason for this problem could be the method of spline interpolation that is applied inside the code. Verscharen et al ${ }^{51}$ mention that the fit method with cubic splines becomes inaccurate for strongly damped solutions. In order to solve the dispersion relation the evaluation of the spline at a complex value is needed, but the value is distant from the real grid points by which the spline is supported.

Further challenges occurred also in the computation of the results of the EFH instability. In order to produce the results we had to use electrons as the first species, so that all results are obtained in electron-based units, and then we transformed the results to proton-units as shown in Figs. 6 and 9. Therefore, we had to reach very low values of $k d_{\mathrm{e}}$, which caused computational issues. Additionally, for very low values of the real and/or imaginary part of the frequency the code easily jumps away from the desired branch of solutions. In general the code goes through the wavenumber interval only forward and one cannot force to the code to stay on a particular solution branch, which means that the code will sometimes jump to the wrong solution branch. This can be somewhat resolved by starting at higher values of $k d_{\mathrm{s}}$ and slowly working back to lower values. These issues will be addressed in our future studies in an attempt to provide a full spectrum of smooth solutions.

Our present results confirm and identify extended abilities of the LEOPARD code, as a powerful tool for the kinetic wave analysis of realistic plasma systems. A great advantage of LEOPARD is the ability to use arbitrary distribution functions, i.e., not being limited to idealized distributions and, therefore, not requiring any pre-derivation of the dielectric tensor for a particular distribution model. Premises are thus provided to integrate such a code with measurements of velocity distributions and describe sufficiently accurate the wave fluctuations detected in parallel by the space probes. In addition, our results in Section III can serve as valuable references to test similar codes, like the recently introduced ALPS (Arbitrary Linear Plasma Solver) $[51$

\section{ACKNOWLEDGMENTS}

EH acknowledges support from the Ruhr-Universität Bochum, and ML acknowledges support in the framework of the projects G0A2316N (FWO-Vlaanderen) and SCHL 201/35-1 (DFG). HF and KS are grateful to the Deutsche Forschungsgemeinschaft, DFG funding the project SCHE334/10-1. We thank the two anonymous referees for their constructive suggestions and comments on the manuscript.

\footnotetext{
${ }^{1}$ P. Astfalk and F. Jenko, J. Geophys. Res. 122, 89 (2017).

${ }^{2}$ M. Maksimovic, I. Zouganelis, J. Y. Chaufray, K. Issautier, E. E. Scime, J. E. Littleton, E. Marsch, D. J. McComas, C. Salem, R. P. Lin, and H. Elliott, J. Geophys. Res. 110, A9 (2005).
} 
${ }^{3}$ I. Zouganelis, N. Meyer-Vernet, S. Landi, M. Maksimovic, and F. Pantellini, Astrophys. J. 626, L117 (2005).

${ }^{4}$ E. Marsch, Liv. Rev. Solar Phys. 3, 1 (2006).

${ }^{5}$ R. Schlickeiser, Cosmic Ray Astrophysics (Springer Science and Business Media, 2002)

${ }^{6}$ M. Lazar, S. Poedts, and M. J. Michno, Astron. Astrophys. 554, A64 (2013).

${ }^{7}$ W. Baumjohann and R. A. Treumann, Basic space plasma physics (Imperial College Press, 1997).

${ }^{8}$ V. Pierrard and M. Lazar, Sol. Phys. 267, 153 (2010).

${ }^{9}$ V. M. Vasyliūnas, J. Geophys. Res.: Space Phys. 73, 2839 (1968).

${ }^{10}$ M. Lazar, S. Poedts, and H. Fichtner, Astron. Astrophys. 582, A124 (2015).

${ }^{11}$ M. Lazar, H. Fichtner, and P. Yoon, Astron. Astrophys. 589, A39 (2016).

${ }^{12}$ S. Olbert, Phys. Magnetosph. 10, 641 (1968).

${ }^{13}$ M. Maksimovic, V. Pierrard, and P. Riley, Geophys. Res. Lett. 24, 9 (1997).

${ }^{14}$ J. Davelaar, M. Mościbrodzka, T. Bronzwaer, and H. Falcke, Astron. Astrophys. 612, A34 (2018).

${ }^{15}$ M. A. de Avillez, J. A. Gervasio, and D. Breitschwerdt, Astron. Astrophys. 616, A58 (2018).

${ }^{16}$ S. Webb, V. N. Litvinenko, and G. Wang, Phys. Rev. STAB 15, 080701 (2012).

${ }^{17}$ I. S. Elkamash and I. Kourakis, Phys. Rev. E 94, 053202 (2016).

${ }^{18}$ M. Lazar and S. Poedts, Astron. Astrophys. 494, 311 (2008).

${ }^{19}$ M. Lazar, V. Pierrard, S. M. Shaaban, H. Fichtner, and S. Poedts, Astron. Astrophys. 602, A44 (2017).

${ }^{20}$ K. Scherer, H. Fichtner, and M. Lazar, Europhys. Lett. 120, 50002 (2017).

${ }^{21}$ K. Scherer, M. Lazar, E. Husidic, and H. Fichtner, Astrophys. J. 880, 118 (2019).

${ }^{22}$ H. Fichtner, K. Scherer, M. Lazar, H. J. Fahr, and Z. Vörös, Phys. Rev. E 98, 053205 (2018).

${ }^{23}$ K. Scherer, H. Fichtner, H. J. Fahr, and M. Lazar, Astrophys. J. 881, 93 (2017).

${ }^{24}$ R. Gaelzer and L. F. Ziebell, J. Geophys. Res. 119, 9334 (2014).

${ }^{25}$ K. Oldham, J. Myland, and J. Spanier, An Atlas of Functions (Springer Science and Business Media, 2000).

${ }^{26}$ C. D. Steenberg and H. Moraal, J. Geophys. Res. 104, A11 (1999).

${ }^{27}$ L. A. Fisk and G. Gloeckler, Space Sci. Rev. 173, 433 (2012).

${ }^{28}$ M. Brambilla, Kinetic Theory of Plasma Waves (Oxford Science Publications, 1998).

${ }^{29}$ P. Astfalk, T. Görler, and F. Jenko, J. Geophys. Res. 120, 7107 (2015).
${ }^{30}$ See https://github.com/pastfalk/LEOPARD for source code of LEOPARD and a manual (last accessed 5 June 2020).

${ }^{31}$ S. P. Gary, Theory of Space Plasma Microinstabilities (Cambridge University Press, 1993).

${ }^{32}$ Š. Štverák, P. Trávniček, M. Maksimovic, E. Marsch, A. N. Fazakerley, and E. E. Scime, J. Geophys. Res. 113, A03 103 (2008).

${ }^{33}$ M. Lazar, R. A. López, S. M. Shaaban, S. Poedts, and H. Fichtner, Astrophys. Space Sci. 364, 171 (2019).

${ }^{34}$ M. Lazar, S. Poedts, and R. Schlickeiser, Mon. Not. R. Astron. Soc. 410, 663 (2011).

${ }^{35}$ R. Helliwell, J. Crary, J. Pope, and R. Smith, J. Geophys. Res. 61, 139 (1956).

${ }^{36}$ R. F. Lutomirski, Phys. Fluids 13, 149 (1970).

${ }^{37}$ D. Verscharen, K. G. Klein, and B. A. Maruca, Living Rev. Sol. Phys. 16, 5 (2019).

${ }^{38}$ L. K. Jian, C. T. Russell, and J. G. Luhmann, Astrophys. J. 701, L105 (2009).

${ }^{39}$ S. T. Nguyen, J. D. Perez, and J. F. Fennell, J. Geophys. Res. 112, A12203 (2007).

${ }^{40}$ M. Lazar, S. M. Shaaban, S. Poedts, and Š. Štverák, Mon. Not. R. Astron. Soc. 464, 564 (2017).

${ }^{41}$ P. Astfalk and F. Jenko, J. Geophys. Res. 121, 2842 (2016).

${ }^{42}$ R. P. Singhal, Elements of Space Physics (PHI Learning Private Limited, 2009).

${ }^{43}$ M. Lazar and S. Poedts, Mon. Not. R. Astron. Soc. 437, 641 (2013).

${ }^{44}$ Y. Maneva, M. Lazar, A. Viñas, and S. Poedts, Astrophys. J. 832, 64 (2016).

${ }^{45}$ R. A. Lopez, M. Lazar, S. M. Shaaban, S. Poedts, P. H. Yoon, A. Viñas, and P. S. Moya, Astrophys. J. 873, L20 (2019).

${ }^{46}$ M. Lazar, S. Poedts, and R. Schlickeiser, Astron. Astrophys. 534, A116 (2011).

${ }^{47}$ R. Gaelzer and L. F. Ziebell, Phys. Plasmas 23, 022110 (2016).

${ }^{48}$ S. M. Shaaban, M. Lazar, P. Astfalk, and S. Poedts, J. Geophys. Res. 123, 1754 (2018).

${ }^{49}$ A. R. Meneses, R. Gaelzer, and L. F. Ziebell, Phys. Plasmas 25, 112901 (2018).

${ }^{50}$ S. M. Shaaban, M. Lazar, R. Lopez, H. Fichtner, and S. Poedts, Mon. Not. R. Astron. Soc. 483, 5642 (2019).

${ }^{51}$ D. Verscharen, K. G. Klein, B. D. G. Chandran, M. L. Stevens, C. S. Salem, and S. D. Bale, J. Plasma Phys. 84, 905840403 (2018). 